\title{
Relationship between Body Mass Index with Menstrual Cycle in Senior High School Students
}

\author{
Rinasyah Ganesh, ${ }^{1}$ Lola Ilona, ${ }^{2}$ Ryadi Fadil ${ }^{3}$ \\ ${ }^{1}$ Faculty of Medicine Universitas Padjadjaran, ${ }^{2}$ Department of Epidemiology and Biostatistics \\ Faculty of Medicine, Universitas Padjadjaran, ${ }^{3}$ Department of Child Health Faculty of Medicine \\ Universitas Padjadjaran/Dr. Hasan Sadikin General Hospital Bandung
}

\begin{abstract}
Background: In recent years, there is an escalation of menstrual disorders in both developing and developed countries. One of the important factors that contribute to menstrual disorders is body fat content. In developing countries like Indonesia, young women in certain cultures get married at average age of 16 years. At the same time, the number of malnourishment among adolescent is increasing. This study was conducted to analyze the relationship between body mass index (BMI) and menstrual cycle in senior high school students.

Methods: This study included 330 girls ages 15-17. This study was conducted in Jatinangor, Sumedang from the period of August-October 2013 using a cross-sectional study. Questionnaires assessing their menstruation and their weight and height were collected after obtaining the respondents consent.The collected data were analyzed using the independent T-test.

Results: Most of the respondents had normal BMI, menstrual interval, amount and duration. There was no relationship between BMI and menstrual interval and menstrual amount ( 0.74 and 0.878 respectively), however there was relationship between BMI and menstrual period $(p=0.036)$.

Conclusions: Menstrual abnormalities among female adolescents are common. Based on this study, BMI seems to be moderately associated with menstrual cycle, especially with menstrual period, although a possibility still remains that body fat affects the menstruation superiorly. [AMJ.2015;2(4):555-60]
\end{abstract}

Keywords: Body mass index, menstrual cycle, senior high school students

\section{Introduction}

Menstruation is a woman's monthly bleeding caused by the reduction of hormones, estrogen and progesterone at the end of the monthly ovarian cycle. ${ }^{1,2}$ When menstruation occurs repeatedly, it is called the menstrual cycle. ${ }^{3}$ The menstrual cycle is important as female reproductive function which prepares the female body for conception and pregnancy. ${ }^{2,3}$

In recent years, there is an escalation of menstrual disorders in both developing and developed countries. However the exact prevalence or incidence of menstrual dysfunction is still very scarce. ${ }^{1}$ Example of menstrual disorders are Amenorrhea, which is lack of menstrual period, dysmenorrheal (painful periods), irregular cycles, heavy and excessive bleeding called menorrhagia. ${ }^{1,3-5}$
One of the important factors that contribute to menstrual disorders is body weight, more specifically body fat content. There are other factors as well, which are divided into external and internal factors. External factors such as caffeine and alcohol consumption, smoking,, stress, occupation, socioeconomic status, and ethnicity. ${ }^{1,3,5}$ While the internal factors are disorders of hormonal imbalance such as thyroid problems and polycystic ovarian syndrome, and body fat content is included in these factors too. ${ }^{6}$

In developing countries, like Indonesia, young women in certain cultures get married at an average age of 16 years. As mentioned before, since the menstrual cycle is important for conception and pregnancy, it is crucial to assess the menstrual cycle in young women. Parallel to this problem, the number of

Correspondence: Rinasyah Ganesh, Faculty of Medicine, Universitas Padjadjaran, Jalan Raya Bandung-Sumedang Km.21, Jatinangor, Sumedang, Indonesia, Phone: +62 85624609741 Email: pottersgirl04@yahoo.com 
malnourished adolescents is increasing as the world population increases. Both in developed and developing countries like Indonesia, socioeconomic status and access to food as well as the influence of media determines malnourishment of a person. Therefore, this study was conducted to analyze the relationship between body mass index (BMI) and the menstrual cycle in senior high school students in Jatinangor, Sumedang, Indonesia from the period of August-October 2013.

\section{Methods}

A cross-sectional study was conducted to 330 senior high school girl students aged 15-17 from a selected school out of the four schools in Jatinangor sub-district Sumedang, Indonesia with the highest number of female students. The students were selected from different classes of the respective school by performing cluster randomization during the period of August-October 2013. After the subjects were excluded based on the exclusion criteria, the remaining three hundred and thirty students were included in the research after meeting the inclusion criteria. The inclusion criteria were aged 15-17 years and already reached menarche. While the exclusion criteria included if they were having hormonal disorders, taking hormonal medications, smoking, and were regular alcohol consumers, also those who had filled the questionnaires incompletely and did not give informed consent.

After the subjects signed the informed consent, questionnaires were distributed to the students to assess their menstrual cycle and explanations were given to assist them in completing the forms. Afterward, the height and weight was measured using a mobile weighing scale and a stadiometer respectively. To categorize the BMI into underweight, normal, overweight and obese, the BMI was plotted on the Centers for Disease Control and Prevention (CDC) chart for "BMI for Age" percentiles.

The menstrual interval/pattern was considered regular, if it is within the range of 21-35 days, and irregular if less than 21 days or more than 35 days. While the menstrual amount was categorized into scanty, normal flow or heavy which was $1-2,3-5,>5$ sanitary towels used respectively per day as revealed by respondents. Then, menstrual duration was classified into short (1-2 days), normal (3-7 days), and prolonged ( $>8$ days) based on the days bleeding occurs.

Furthermore, the collected data were statistically analyzed using Independent T-test. Analysis is performed using the menstrual interval, menstrual amount, and menstrual period among underweight, normal, overweight and obese students. This study was approved by the Health Research Ethical Committee of Dr. Hasan Sadikin General Hospital. This study was part of a bigger research called Jatinangor Cohort.

\section{Results}

The characteristic of the research subjects shows that all the variables were not normally distributed except for height (Table 1).

The BMI of the respondents were classified into underweight, normal, overweight, and obese. It showed that the number of respondents with normal weight was more than the underweight and overweight/obese respondents. It also showed subjects with regular menstrual interval were more than the subjects with irregular menstrual interval. Besides, there were a significant number of subjects with scanty menstruation compared to subjects with heavy menstruation. It was

Table 1Characteristic of Senior High School Students in Jatinangor

\begin{tabular}{|c|c|c|}
\hline Variables & Mean & p-value \\
\hline Age & 15.8 & $<0.01$ \\
\hline Height $(\mathrm{cm})$ & 152.6 & $0.200 *$ \\
\hline Weight & 47.3 & $<0.01$ \\
\hline Body Mass Index (BMI) & 20.3 & $<0.01$ \\
\hline Menarche Age & 12.6 & $<0.01$ \\
\hline Days Bleeding Occurs & 6.7 & $<0.01$ \\
\hline Number of Sanitary Towels Used per day & 2.8 & $<0.01$ \\
\hline
\end{tabular}

Note: *indicates normal distribution using Kolmogorov-Smirnov normality test 
Table 2 Frequency of BMI, Menstrual Interval, Menstrual Amount \& Menstrual Period Status

\begin{tabular}{lccc}
\hline & $\begin{array}{c}\text { Criteria } \\
\text { (n=330) }\end{array}$ & f & $\%$ \\
\hline Body Mass Index (BMI) & Underweight & 39 & 11.8 \\
& Normal & 257 & 77.9 \\
& Overweight & 29 & 8.8 \\
& Obese & 5 & 1.5 \\
Menstrual Interval & Short Irregular & 52 & 15.8 \\
& Regular (Normal) & 247 & 74.9 \\
& Prolonged Irregular & 31 & 9.4 \\
Menstrual Amount & Scanty & 130 & 39.4 \\
& Normal Flow & 197 & 59.7 \\
& Heavy & 3 & 0.9 \\
Menstrual Period & Short & 0 & 0.0 \\
& Normal & 286 & 86.7 \\
& Prolonged & 44 & 13.3 \\
\hline
\end{tabular}

also noted that among the three hundred and thirty subjects, none was reported of having a short menstrual duration. Most of them reported of having a normal menstrual period and the rest reported of having prolonged menstruation (Table 2).

The majority of subjects reported of having a regular menstrual interval compared to irregular interval. On top of that, the percentage of obese students having either short or prolonged menstrual interval was higher compared to other BMI category subjects (Figure 1).

The analysis performed between BMI and menstrual interval revealed that there was no significance with $p$ value $=0.074$. It also found that the mean BMI for subjects with short irregular menstrual interval was 20.4158 while the mean of BMI for subjects with regular and prolonged irregular was 20.2610.

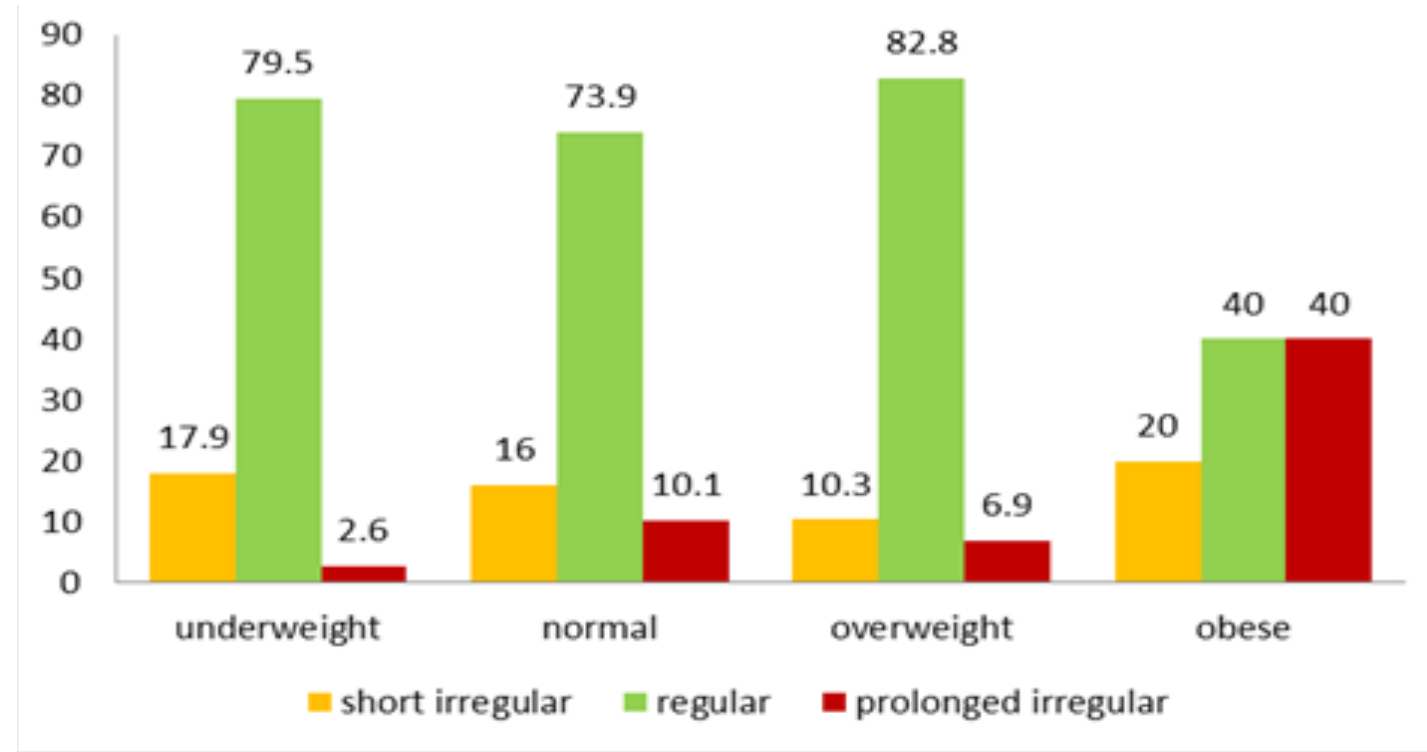

Figure 1 Distribution of Different Menstrual Intervals among Different Categories of BMI 


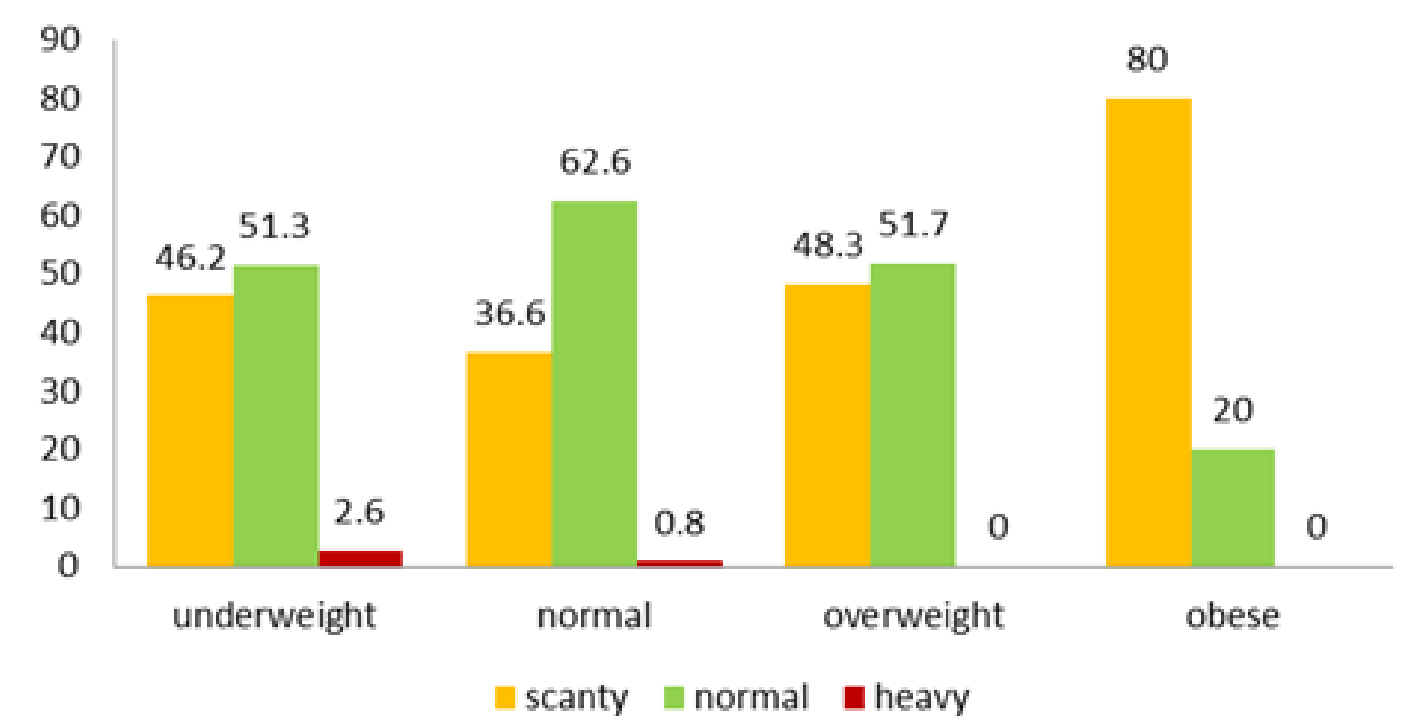

Figure 2 Distribution between Different Menstrual Amounts with Different Categories of
BMI

Furthermore, higher percentage of obese subjects complained of abnormal menstrual amount, followed concurrently by underweight and overweight subjects and finally by normal weight subjects. Besides, it discovered that very few subjects reported of having a heavy menstruation, in this case 3,4 $\%$ with none of overweight/ obese subjects reported so (Figure 2). The analysis revealed that $p$ value $=0.878$ which indicated there was no significance between BMI and menstrual amount. It also revealed that the mean of BMI for subjects with scanty menstrual amount was 20.2524 and 20.3069 for subjects with normal and heavy menstruation.

None of the of the respondents revealed of having a short menstrual period, with majority falling into having a normal menstrual period,

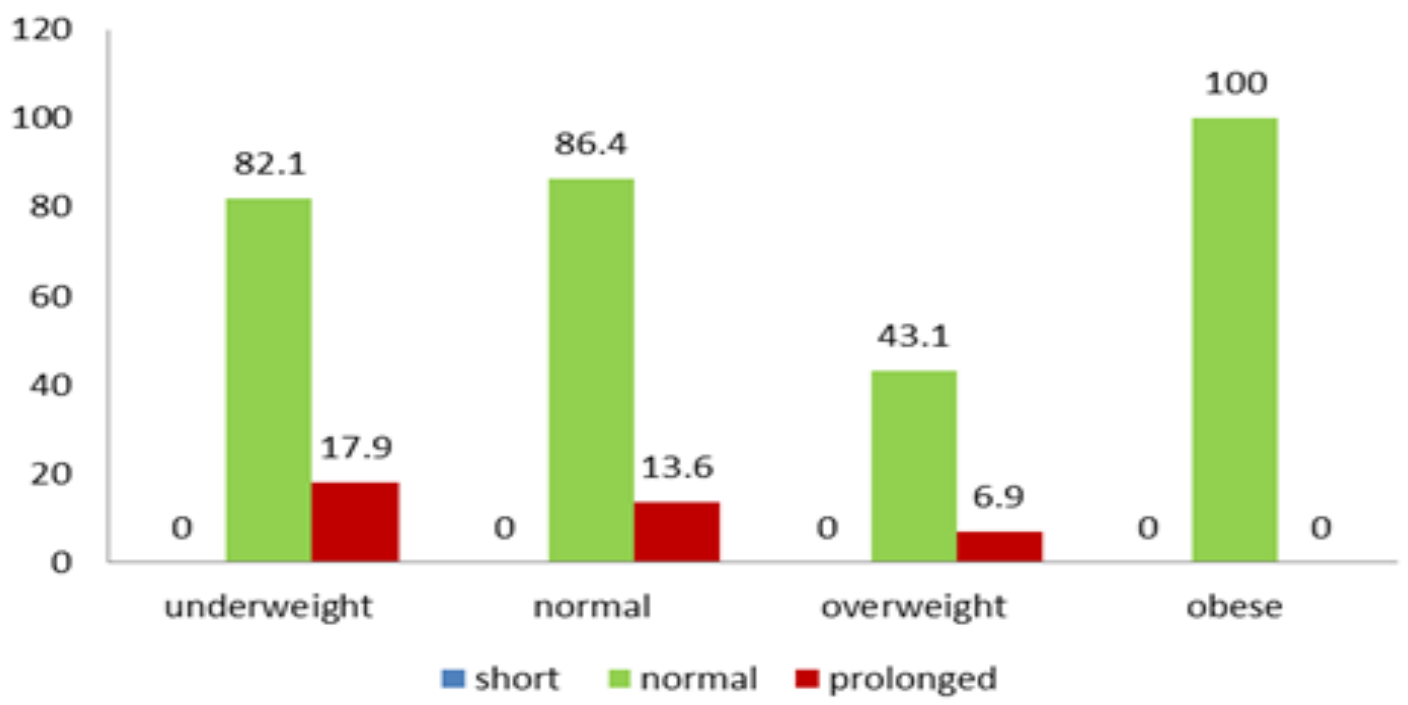

Figure 3 Distribution of Different Length of Menstrual Period with Different Categories of BMI 
followed by a few subjects into prolonged menstrual period. Subjects with prolonged period, were mainly reported by underweight subjects (Figure 3). The relationship between BMI and menstrual period exposed that $p$ value $=0.036$ indicating the moderate significance. The subjects with a normal menstrual period had a mean BMI of 20.3948 and for subjects with prolonged menstrual period was 19.5743 . This evidently showed the difference of BMI mean between the normal and prolonged menstruation, which clearly signifies the relationship.

\section{Discussion}

The BMI is a statistical measure which compares an individual's height and weight. It was used as diagnostic tool in this study to identify the nutritional status within a population due to its ease of calculation. However, BMI does not take into account many factors like frame size, muscularity, body fat, bone, cartilage, and water weight.7 Additionally, body fat content is one of the factors that affect menstruation by altering the hormones associated with menstruation. This could explain why the relationship between BMI and menstrual cycle was not so dominant since BMI did not accurately measure body fat. Evidently, not all of the previous studies supported the association of BMI and menstrual disorderssince there were other factors that influenced the menstrual cycle.

In a study conducted in Singapore in 2009 to detect menstrual disorders in adolescent girls, it found that with the increase of BMI, there is significant increase in the prevalence of oligomenorrhea, whereas polymenorrhea is more prevalent in girls with low BMI.8 Similar to this study, a study conducted in India among older girls studying in a Medical Teaching Institution found correlation between irregular cycles and high BMI. ${ }^{9}$

Contrary to above mentioned studies, a study conducted in Japan among female workers in 2011, determined that stress is the major factor related to menstrual cycle irregularities and menstrual pain. In that study, factors such as smell of cigarettes, stress, age, BMI, smoking habits and sleeping hours were investigated if they were associated with menstrual disorders. Furthermore, the factors that showed significance are stress, smell of cigarettes, age and smoking habits. Stress activates the release of corticotrophin in the nervous system causing menstrual cycle irregularities and interferes with a mixture of endocrine profiles particularly gonadotropin and estrogen secretion. ${ }^{10}$

The BMI in this study showed no significance. ${ }^{10}$ Supporting this, another study conducted in Malaysia in 2006 among teenagers, showed that BMI is not one the factors associated with menstrual disorders such as abnormal period of menstrual flow, heavy menstrual flow, irregular menstrual cycle and dysmenorrheal however BMI is associated with premenstrual syndrome, that are more commonly among overweight or obese subjects. Factors that are associated with menstrual disorders are smoking, followed by suicidal behavior, depression, anxiety which may be attributed to increased stress, then menarche within 2 years, age and ethnicity. It also finds that dieting behavior (eating less to lose weight) and those who are physically inactive affect the menstrual cycle as well. ${ }^{11}$

Thus, based on the above mentioned sources, factors such as stress, emotions, smoking, ethnicity, age, eating habits or disorders, and physical activity could be the related factors affecting the respondents with menstrual disorders in this study, besides the possibility of having pathological conditions such as polycystic ovarian syndrome.

One of the limitations of this study was that there were not enough underweight, overweight or obese subjects to assess the menstruation pattern among adolescents and therefore it was unable to accurately detect the various menstrual disorders. This was due to the lack of underweight or overweight population present in Jatinangor compared to Bandung city.

The second limitation was that the results were based on the adolescents' own reports of their behaviors, such as smoking and alcohol consumption. There was a possibility that some respondents might have not reported smoking and alcohol consumption to avoid judgment or a bad impression.

It was also found that a few students, especially those in classes with poor academic levels, might not have been able to understand the questions. Even though, they were assisted by the interviewers, still some invalid answers were received. However, these answers were not omitted from the analysis as it was difficult to determine which were invalid.

Finally, it can be concluded that menstrual abnormalities among female adolescents are common. ${ }^{11}$ Based on this study, BMI seems to be moderately associated with menstrual cycle, especially with menstrual period, although a possibility still remains 
that body fat does affect the menstruation superiorly. A research should be conducted in the future about the influence of body fat on menstruation using an accurate measurement of body fat. Besides, there are many factors that need to be discovered for prompt diagnosis and treatment to prevent infertility and complication later in life, especially where there are women in certain cultures who get married at an early age. Moreover, health education on menstrual problems should be given to female adolescents when they approach the reproduction age and also to their parents; especially the mothers who are the main source of knowledge for their daughters, as well as regular screening for menstrual abnormalities should be performed.

\section{References}

1. Goldman MB, Hatch MC, editors. Menstruation and menstrual disorders. Women \& Health. California,USA: Acad. Press. 2004. p. 99-113.

2. Hall JE. Female physiology before pregnancy and female hormones. In: Guyton and Hall Textbook of Medical Physiology. 12th ed. Pennsylvania, USA: Saunders Elsevier. 2011. p. 987-1002.

3. Lawrence M. Nelson MD. Menstruation and menstrual cycle fact sheet. Womenshealth. gov. 2009 [cited 2013 January 12]; Available from: http://www.womenshealth.gov/ publications/our-publications/factsheet/menstruation.html.
4. Harlow SD, Campbell OMR. Epidemiology of menstrual disorders in developing countries : a systematic review. BJOG. 2004;111(1):6-16.

5. Liu Y, Gold EB, Lasley BL, Johnson WO. Factors affecting menstrual cycle characteristics. Am J Epidemiol. 2004; 160(2):131-40.

6. Panay N, Dutta R, Ryan A, Broadbent JAM. Abnormal Bleeding. Obstetrics and Gynaecology. Pennsylvania, USA: Elsevier. 2004. p. 1-10.

7. Chauhan M, Kala J. Relation between dysmenorrhea and body mass index in adolescents with rural versus urban variation. J Obstet Gynaecol India. 2012; 62(4):442-5.

8. Agarwal A, Venkat A. Questionnaire study on menstrual disorders in adolescent girls in Singapore. J Pediatr Adolesc Gynecol. 2009;22(6):365-71.

9. Deshpande H, Burute SB, Dahiya P. Relationship of body mass index and body fat percentage with menstrual cycle pattern in adolescents. Int J Pharm. 2013;4(4):114-7.

10. Nohara M, Momoeda M, Kubota $T$, Nakabayashi M. Menstrual cycle and menstrual pain problems and related risk factors among Japanese female workers. Ind health. 2011;49(2):228-34.

11. Lee L, Chen P, Lee K, Kaur J. Menstruation among adolescent girls in Malaysia: a cross-sectional school survey. Singapore Med J. 2006;47(10):869-74. 